Article

\title{
Capacitive Emulation Using Predictive Current Control in LCL-Filtered Grid-Connected Converters to Mitigate Grid Current Distortion
}

\author{
Jose Miguel Espi ${ }^{(\mathbb{D})}$ and Jaime Castello * (1) \\ Department of Electrical Engineering, University of Valencia, Avd. Universitat S/N, \\ 46100 Burjassot-Valencia, Spain; jose.m.espi@uv.es \\ * Correspondence: jaime.castello@uv.es; Tel.: +34-963-543-504
}

Received: 23 May 2018; Accepted: 30 May 2018; Published: 1 June 2018

\begin{abstract}
This paper presents an improvement of the capacitive emulation (CE) method to reduce the line current distortion caused by grid-tied LCL-filtered converters. In these cases, the grid voltage is applied to the LCL's capacitor, which generates a distorted capacitor current that pollutes the line. The CE method consists in controlling the converter currents instead of the grid currents, while the converter generates a copy of the distorted capacitor current, so that both the copy and the distorted currents cancel each other in the grid. Therefore, we can say that the converter emulates a negative capacitance connected to the grid, while at the same time delivers its active and reactive powers at the fundamental frequency. The $\mathrm{CE}$ method is achieved by adding an estimation of the distorted capacitor current to the converter current reference. However, an effective capacitive emulation requires a current control capable of accurately tracking all harmonics added to the current reference. In this sense, this paper proposes the use of a new integral predictive current control (IPCC), a dead-beat type of control that ensures a constant closed-loop group delay in a wide bandwidth. Unlike a PI control where the closed-loop delay varies with the frequency of the tracked harmonic, the constant control delay of the IPCC can be effectively compensated with a buffer-based advanced current reference. The effectiveness of the proposed CE method with IPCC control to reduce the total harmonic distortion (THD) of the line currents has been proved experimentally on a $10 \mathrm{kVA}$ transformerless grid-connected three-phase inverter.
\end{abstract}

Keywords: capacitive emulation (CE); integral predictive current control (IPCC); converter current feedback (CCF); grid current feedback (GCF); total harmonic distortion (THD)

\section{Introduction}

Usually, two different current control strategies are used for grid-connected converters with LCL filter [1-4]: to sense and control the converter currents, i.e., converter current feedback (CCF), or to sense and control the grid currents directly, i.e., grid current feedback (GCF).

The main drawback of the CCF scheme is that, even if the controlled converter currents are achieved sinusoidal, the line currents are distorted by the LCL's capacitor currents. Hence, the GCF [5-8] emerged to solve the distortion problem of the CCF. It is usually implemented using a proportional resonant (PR) control with enough bandwidth and number of resonances to ensure the rejection of the grid current harmonics, resulting in line currents with low THD factor. The GCF however has a higher loop gain resonance peak compared to the CCF [3,9], which must be damped by means of active damping (AD) techniques $[5,6,10,11]$ to achieve a large control bandwidth, thus increasing complexity. In contrast, in the CCF case, the loop resonance can be damped by simply adding a small resistor in series with the LCL capacitors. 
To solve the distortion problem of the CCF scheme, Espi et al. [12] proposed the capacitive emulation (CE) technique. The main idea is to add the distorting capacitive currents to the controlled inverter currents, so that both cancel each other out in the grid. That is, the converter emulates a negative capacitance connected to the grid while delivering the active and reactive powers at the fundamental frequency. It was proven in [12] that the $\mathrm{CE}$ method can compensate current harmonics up to the $L_{2}-C$ resonance (where $L_{2}$ and $C$ are defined in Figure 1) giving better results than the GCF techniques in terms of $\mathrm{THD}_{i}$. The CE method consists on:

1. Estimate the capacitive current disturbance as explained in [12], and feed-forward it to the current reference. This estimation $\left(i_{c}^{d q}\right.$ in Figure 1$)$ has to be ahead to compensate for the closed-loop delay of the current control.

2. Implement a current control capable to track the reference harmonics added. Therefore, the goal is to achieve a nearly constant closed-loop frequency response at the compensating bandwidth.

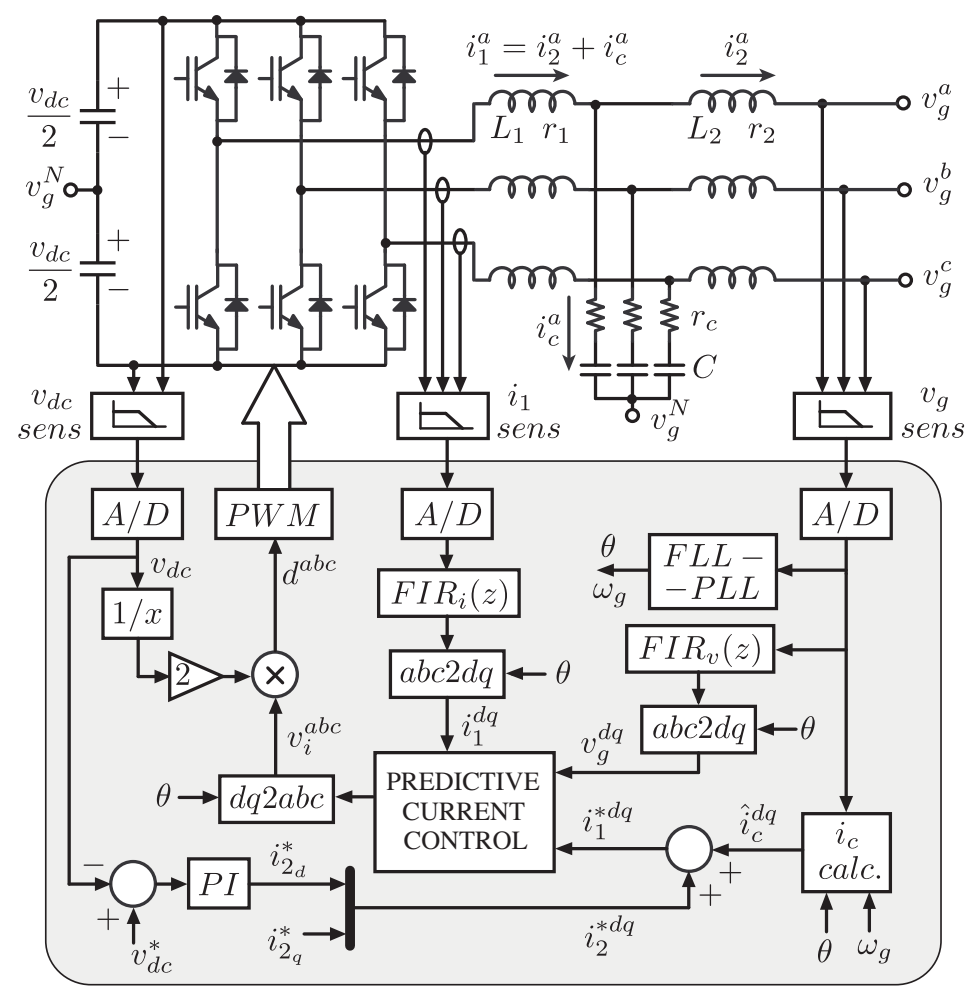

Figure 1. Transformerless three-phase grid-connected inverter with LCL filter, predictive current control and harmonics cancellation strategy.

Espi et al. [12] proposed an estimation of the capacitive current a constant number of samples ahead, resulting an effective method to compensate any constant control delay. However, the delay compensation in [12] was not fully effective for all harmonics, as the PI control used did not exhibit a constant group delay in closed-loop for all frequencies. To overcome this drawback, present paper proposes the use of a new predictive current control to implement the CE method, which features a constant group delay (a constant number of samples) in closed-loop. This improves the CE performance, as shown experimentally below. The proposed predictive control is based in [13], which is modified by adding one integrator to remove the steady-state current error at the fundamental harmonic while preserving the closed-loop dead-beat performance, thus resulting an integral predictive current control (IPCC). We want to emphasise that this is not a finite-state model predictive control (FS-MPC) [14,15]. While the IPCC outputs a conventional PWM signal, the FS-MPC selects the voltage vector instead to minimise a cost function (usually the norm or the quadratic value of the control error) 
in a given horizon. As the FS-MPC requires more time to calculate, it is solved at lower sampling frequency, and this impedes the high frequency current harmonics generation required by the $\mathrm{CE}$ method (up to 17th-19th). Moreover, the cost function minimisation of the FS-MPC does not guarantee a constant closed-loop group delay.

\section{Proposed Integral Predictive Current Control}

The LCL filter in Figure 1 is represented in the $\alpha \beta$ frame by two decoupled circuits, as shown in Figure 2, where $v_{g}^{\alpha \beta}$ and $v_{i}^{\alpha \beta}$ are the grid voltage and the averaged inverter voltage, respectively. Inductors models $Z_{1}$ and $Z_{2}$ include their inductances $L$, series resistances $r$ and iron core losses resistances $R_{F e}$. Resistance $r_{c}$ accounts for the capacitor series resistance and any added damping resistance.

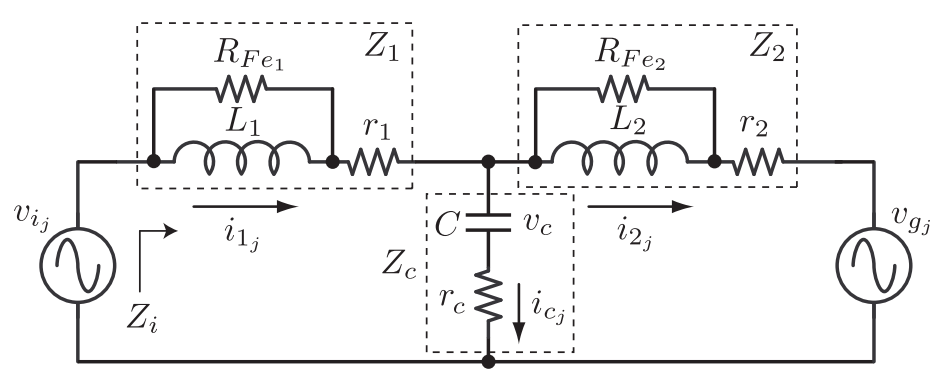

Figure 2. Model of the LCL filter in the $\alpha \beta$ reference frame $(j=\{\alpha, \beta\})$.

Figure 3 shows the LCL filter model in the synchronous reference frame. For frequencies below the resonance $L_{2}-C$, the circuit in Figure 3 can be simplified, as shown in Figure 4a, where $C \omega_{g} v_{g q} \ll i_{1_{d}} \approx i_{2_{d}}$ and $L_{2} C \omega_{g}^{2} \ll 1$ has been taken into account. Moreover, the circuit in Figure $4 \mathrm{a}$ can be simplified if the inverter voltages are calculated as

$$
\begin{aligned}
& v_{i_{d}}=v_{g_{d}}-L \omega_{g} i_{1_{q}}+v_{i c_{d}} \\
& v_{i_{q}}=v_{g_{q}}+L \omega_{g} i_{1_{d}}-r_{2} C \omega_{g} v_{g_{d}}+v_{i c_{q}}
\end{aligned}
$$

resulting the decoupled circuit shown in Figure $4 b$, where $v_{i c_{j}}(j=\{d, q\})$ represents the averaged voltage applied to an equivalent inductance $L=L_{1}+L_{2}$ and resistance $r_{L}=r_{1}+r_{2}$.

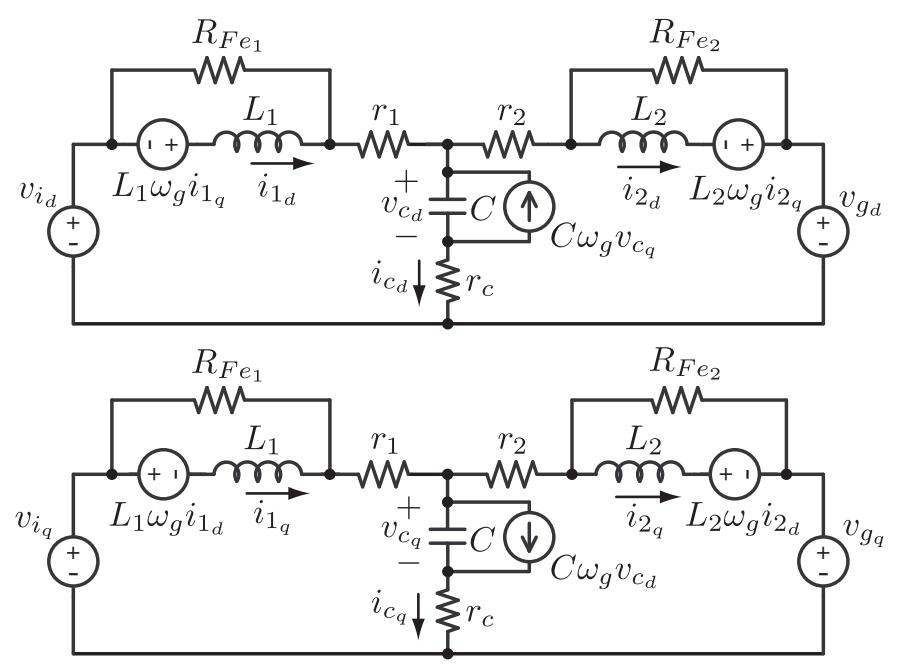

Figure 3. Model of the LCL filter in the $d q$ reference frame. 


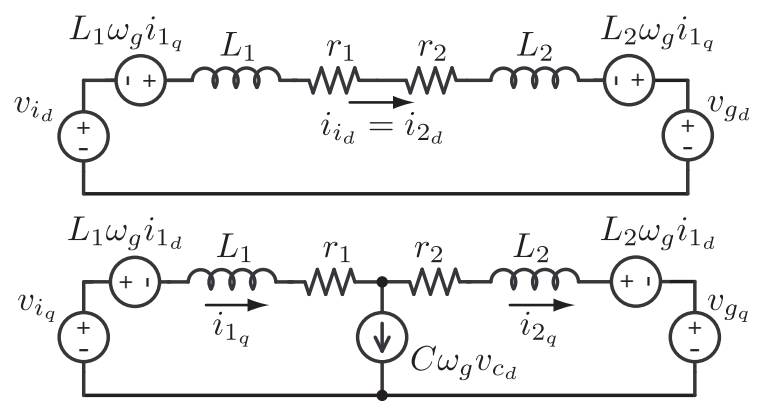

(a)

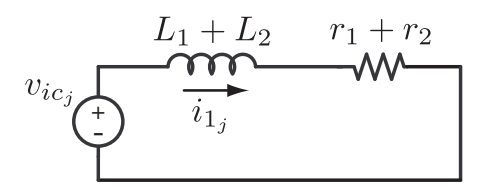

(b)

Figure 4. Low frequency $d q$ model of the LCL filter: (a) with cross-coupling terms; and (b) simplified by using feed-forward control technique $(j=\{d, q\})$.

Considering the resulting model in Figure $4 \mathrm{~b}$ and the control delay of one sampling period $T$ (Figure 5a), the discretized equation for $i_{1_{j}}$ is

$$
i_{1_{j}}(k)=\beta \cdot i_{1_{j}}(k-1)+\frac{T}{L} v_{i c_{j}}(k-2)
$$

where $\beta=e^{-\frac{r_{L}}{L} T} \lesssim 1$

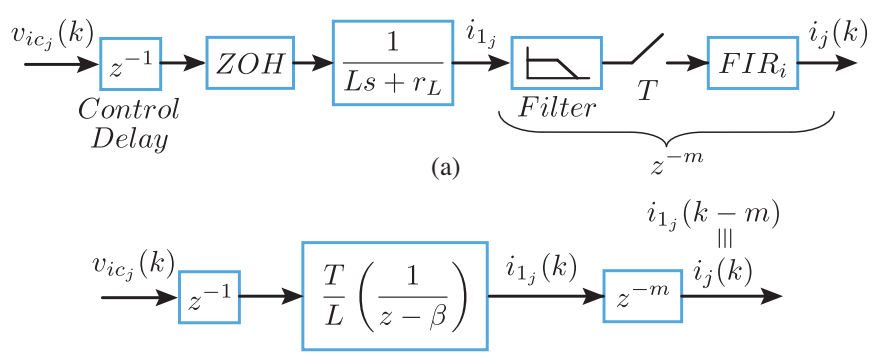

(b)

Figure 5. Plant model with: (a) anti-aliasing filter, control and FIR delays; and (b) equivalent model.

Figure $5 \mathrm{a}$ includes the anti-aliasing filtering of the inverter currents. These filters are designed to have an almost constant group delay, so that they can be modelled as a pure delay.

The predictive control performs better when the total delay of the acquired currents is an integer multiple of $T$ [13], since the current observers can only predict an integer number of samples ahead. Thus, a FIR filter is added as

$$
\operatorname{FIR}_{i}(z)=1-\delta+\delta z^{-1}
$$

with $\delta \in[0,1]$, which adds a fractional delay $\delta T$ to get an overall acquisition delay of $m$ samples (Figure $5 \mathrm{~b}$ ). Defining the acquired current as $i_{j}(k) \equiv i_{1_{j}}(k-m)$, the discrete equation for $i_{j}$ becomes

$$
i_{j}(k)=\beta \cdot i_{j}(k-1)+\frac{T}{L} v_{i c_{j}}(k-m-2) .
$$


By advancing $m+2$ samples in Equation (4) and letting $i_{j}^{*}(k) \equiv i_{j}(k+m+2)$ be the current reference, the dead-beat control law shown in Figure 6a is derived as

$$
v_{i c_{j}}(k)=\frac{L}{T}\left[i_{j}^{*}(k)-\beta \cdot \hat{i}_{j}(k+m+1)\right]
$$

where $\hat{i}_{j}(k+m+1)$ is the $m+1$ forward estimation of $i_{j}$ obtained by programming $n=m+1$ cascaded Luenberger observers. Each observer is implemented as depicted in Figure $6 \mathrm{~b}$, where $L_{o} \in(0, \beta)$ is the observer gain. Notice that the settling time for $i_{j}$ will be $m+2$ samples, but it is just two samples for the actual current $i_{1}$. Thus, the current control behaves ideally as $z^{-2}$ in closed-loop.

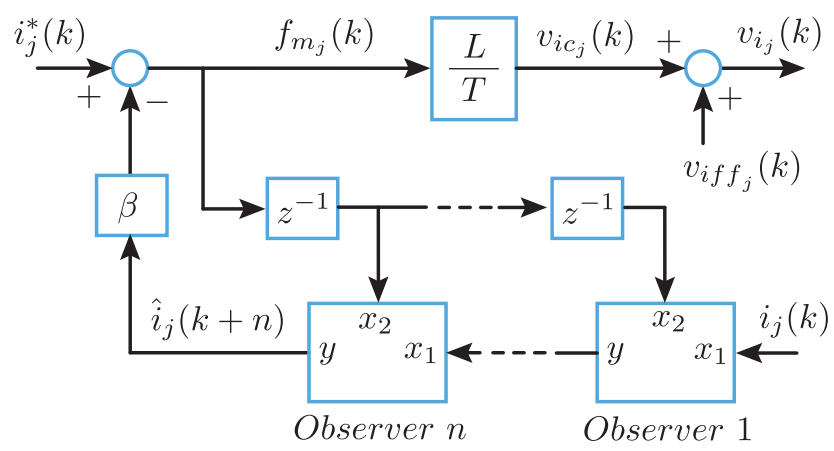

(a)

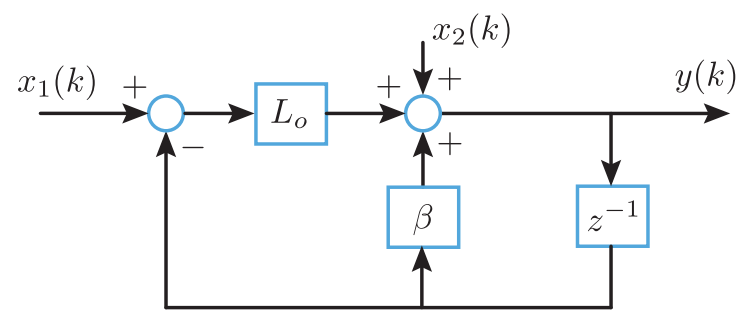

(b)

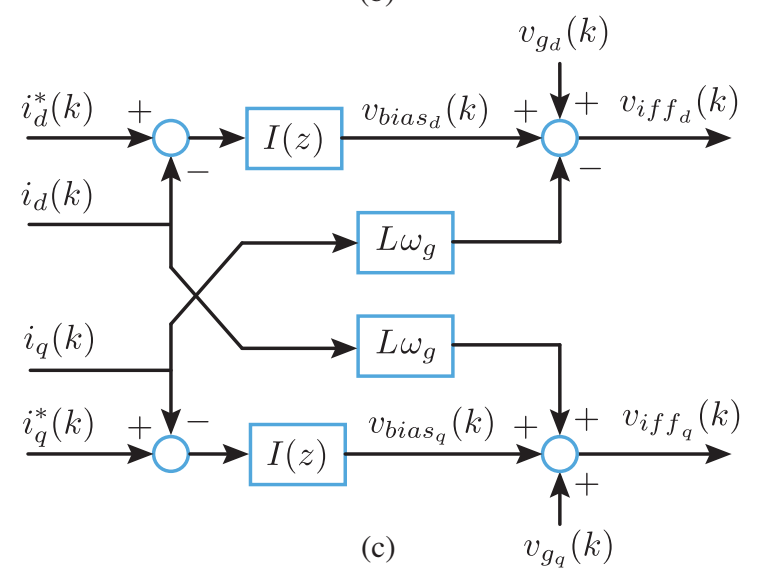

Figure 6. Integral Predictive Current Control (IPCC): (a) predictive control $(j=\{d, q\})$; (b) observer implementation for one sample forward prediction.; and (c) feed-forward term calculation.

One major problem of predictive current controls is their non-negligible steady-state error, as they present a finite dc-gain. Here, the current error increases as the control parameters $L$ and $\beta$ in Figure 6 differs from their actual counterparts in the filter. This problem was solved in [13] by a time-varying control, in which the value of $\beta$ was self-tuned by an additional loop to remove the current error at any operating condition. In this paper, a simpler and more effective approach, is suggested consisting in using an integrator working in parallel with the predictive compensator while the control parameter $\beta$ 
is kept constant. The decoupling terms in Equation (1) and the integrators outputs are gathered under the feed-forward terms $v_{i f f_{j}}$ shown in Figure $6 \mathrm{c}$, being the integrator $I(z)=k_{I} T z /(z-1)$. The constant term $r_{2} C \omega_{g} v_{g_{d}}$ in Equation (1) is not programmed, since it is automatically generated by the $q$-side integrator and included in $v_{b i a s_{q}}$.

Figure 7 shows the typical equivalent block diagram of a current control, where $G_{c}$ is the equivalent compensator and $G_{p}$ the equivalent prefilter. In the case of the predictive control presented in [13], the transfer functions of $G_{c}$ and $G_{p}$ are dependent on the number of observers $n$, being

$$
\begin{aligned}
& G_{c}(z)= \begin{cases}\frac{\frac{L}{T} \beta L_{o} z}{z+L_{o}} \quad(\text { if } n=1) \\
\frac{\frac{L}{T} L_{o}^{2} \beta z^{2}}{z^{2}+\left(2 L_{o}-\beta\right) z+L_{o}^{2}} & (\text { if } n=2)\end{cases} \\
& G_{p}(z)= \begin{cases}\frac{z-\left(\beta-L_{o}\right)}{\beta L_{o} z} & (\text { if } n=1) \\
\frac{1}{\beta}\left(\frac{z+L_{o}-\beta}{L_{o} z}\right)^{2} & (\text { if } n=2) .\end{cases} \\
& \stackrel{i_{j}^{*}}{\rightarrow} G_{p}(z) \rightarrow G_{c}(z) \stackrel{v_{i c_{j}}}{\longrightarrow} \underset{\frac{T}{L} \cdot \frac{z^{-(m+1)}}{z-\beta}}{G_{s}(z)} \stackrel{i_{j}}{\longrightarrow}
\end{aligned}
$$

Figure 7. Equivalent block diagram with prefilter $(j=\{d, q\})$.

It was proven in [13] that, for all $n$ and assuming $\beta \approx 1$, the dc-gain of the compensator is

$$
G_{c_{0}}=G_{c}(z=1) \approx \frac{L}{T}\left(\frac{L_{o}}{n+L_{o}}\right)
$$

and therefore the open-loop gain crossover frequency is

$$
\omega_{c}=\frac{G_{c_{0}}}{L} \approx \frac{1}{T}\left(\frac{L_{o}}{n+L_{o}}\right) .
$$

In this paper, the proposed IPCC adds the integrator contribution, as can be seen in Figure 8a, which can be simplified as in Figure $8 b$, leading again to an equivalent compensator $G_{c}^{\prime}$ and prefilter $G_{p}^{\prime}$. As the new compensator is $G_{c}^{\prime}=G_{c}+I$, it will present a zero at the frequency

$$
\omega_{z}=\frac{k_{I}}{G_{c_{0}}} \approx \frac{\alpha}{T}\left(\frac{n+L_{0}}{L_{o}}\right)
$$

where the integrator gain has been rewritten as $k_{I}=\alpha L / T^{2}$. The zero's frequency $\omega_{z}$ has to be designed below $\omega_{c}$ to keep the IPCC's dead-beat properties. Taking $\omega_{c} 50$ times $\omega_{z}$, the design equation for the integrator normalized gain yields

$$
\alpha=\frac{1}{50}\left(\frac{L_{o}}{n+L_{o}}\right)^{2} .
$$

If an $\alpha$ value higher than that recommended by Equation (11) is used (i.e., a faster integrator), the dead-beat properties could be lost, hence presenting a larger settling time. However, if the recommended $\alpha$ is used, the control behaves as a two samples delay as expected from the predictive law. 


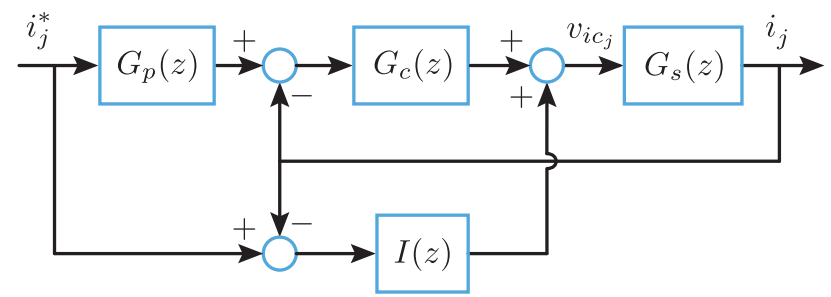

(a)

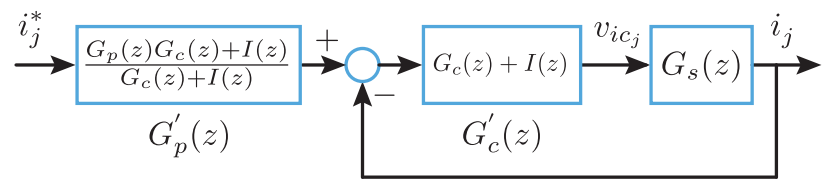

(b)

Figure 8. Proposed IPCC control: (a) block diagram; and (b) simplified block diagram in a prefilter $G_{p}^{\prime}(z)$ and compensator $G_{c}^{\prime}(z)$ form $(j=\{d, q\})$.

\section{Experimental Results}

A $10 \mathrm{kVA}$ transformerless three-phase back-to-back converter was built to assess the improved CE strategy with IPCC and to compare it with most common CCF and GCF strategies, including the basic CE with PI control described in [12]. Two Intelligent Power Modules IPM-PS22A79 from Mitsubishi were used to build the rectifier and the inverter, respectively. The inverter solves the current control and the CE method, while the rectifier regulates the dc-bus voltage to $800 \mathrm{~V}$. Four $470 \mu \mathrm{F} / 450 \mathrm{~V}$ capacitors were used to build the dc-bus capacitance, reaching up to $900 \mathrm{~V}$. Hall effect sensors LTS 15-NP were used for current sensing, and LV25-P for the grid voltage and dc-voltage sensing. Table 1 gives the parameters of the inverter side LCL filter. The damping coefficient $\zeta=0.74$ of all anti-aliasing filters results in a minimum variation of the group delay [16], minimizing distortion of acquired currents. The control was programmed in a RX630 Renesas floating point microcontroller. An FLL-PLL [17] solves the grid synchronization. The FLL measures the grid frequency $\omega_{g}$ needed to estimate the capacitive distortion $\hat{i}_{c}^{d q}$ as explained in [12], to adapt the resonant frequencies $\omega_{h}$ of the PR control in Equation (16) and to calculate the decoupling terms in Equation (1).

Table 1. Plant parameters.

\begin{tabular}{lccc}
\hline Description & Variable & Value & p.u. \\
\hline Nominal power & $P$ & $10 \mathrm{kVA}$ & 1.0 \\
Grid frequency & $\omega_{g}$ & $2 \pi 50 \mathrm{rad} / \mathrm{s}$ & 1.0 \\
Grid voltage (per line) & $v_{g}$ & $230 \mathrm{~V} \mathrm{rms}$ & 1.0 \\
Switching frequency & $f_{s w}$ & $10 \mathrm{kHz}$ & 200 \\
DC-bus voltage & $v_{d c}$ & $800 \mathrm{~V}$ & 2.46 \\
Inverter side inductance & $L_{1}$ & $1 \mathrm{mH}$ & 0.0198 \\
Inverter side core losses resistance & $R_{F e_{1}}$ & $1.3 \mathrm{k} \Omega$ & 81.92 \\
Inverter side series resistance & $r_{1}$ & $30 \mathrm{~m} \Omega$ & 0.0019 \\
Trans./Diodes eq. losses resistance & $R_{s w}$ & $0.32 \Omega$ & 0.0202 \\
Grid side inductance & $L_{2}$ & $180 \mu \mathrm{H}$ & 0.0036 \\
Grid side core losses resistance & $R_{F e_{2}}$ & $350 \Omega$ & 22.05 \\
Grid side series resistance & $r_{2}$ & $120 \mathrm{~m} \Omega$ & 0.0076 \\
LCL capacitance & $C$ & $19 \mu \mathrm{F}$ & 0.0947 \\
LCL capacitor series resistance & $r_{c}$ & $30 \mathrm{~m} \Omega$ & 0.0019 \\
Anti-aliasing filter (cutoff freq.) & $f_{a}$ & $5 \mathrm{kHz}$ & 100 \\
Anti-aliasing filter (damping) & $\zeta$ & 0.74 & 0.74 \\
\hline
\end{tabular}


The programmed IPCC parameters are listed in Table 2. Parameter $L_{o}=0.67$ sets the bandwidth to $f_{c}=800 \mathrm{~Hz}$ according to Equation (9). The integrator gain is designed using Equation (11), yielding $k_{I}=594$. As the anti-aliasing filter delay at $50 \mathrm{~Hz}$ is $t_{d} \approx 47 \mu \mathrm{s}$, the FIR's fractional delay is $\delta=\left(T-t_{d}\right) / T=0.06$ to complete a sensing delay of $m=1$ sample.

Table 2. IPCC control parameters.

\begin{tabular}{lcc}
\hline Description & Variable & Value \\
\hline Sampling period & $T$ & $50 \mu \mathrm{s}$ \\
Gain crossover frequency & $\omega_{c}$ & $2 \pi 800 \mathrm{rad} / \mathrm{s}$ \\
Number of cascaded observers & $n$ & 2 \\
Observer gain & $L_{o}$ & 0.67 \\
Integrator gain & $k_{I}$ & $594 \Omega \cdot \mathrm{s}^{-1}$ \\
Programmed $\beta$ & $\beta$ & 0.98 \\
FIR fractional delay & $\delta$ & 0.06 \\
\hline
\end{tabular}

The first experimental result, shown in Figure 9, investigates the closed-loop performance of the proposed IPCC, a PI and a PR controls in a CCF scheme, that is, the ability of these controls to track reference harmonics. These results were obtained by programming the current references

$$
\begin{aligned}
& i_{d}^{*}=I_{0} \cdot \cos \left(\theta_{0}\right)+I \cdot \cos \left[h \cdot\left(\theta+\frac{\pi}{2}\right)\right] \\
& i_{q}^{*}=-I_{0} \cdot \sin \left(\theta_{0}\right)+I \cdot \sin \left[h \cdot\left(\theta+\frac{\pi}{2}\right)\right]
\end{aligned}
$$

with $I_{0}=5 \mathrm{~A}, I=3 \mathrm{~A}$ and sweeping for the harmonics $h=\{1,2, \ldots, 49\}$. A Power and Quality Analyzer CA8384B from Chauvin Arnoux was used to measure the amplitude and phase $\phi$ of the resulting harmonic on $i_{1}$. As the reference harmonic in Equation (12) is in phase with the grid voltage and the analyser measures harmonic phases referred to the fundamental current, both fundamental voltage and current have to be perfectly in phase in order to get delay measurements $\phi$ referred to the reference harmonic. This is achieved by adjusting $\theta_{0}$ before starting the measurements. The group delay was approximated by $G_{d}(h)=\frac{-\phi}{\omega_{g} h T}$ samples, being $\phi$ the measured closed-loop phase in radians.

Table 3 gives the PI and PR control parameters. The PI compensator is

$$
G_{c}(z)=k_{p}+\frac{k_{i} T z}{z-1}
$$

where $k_{p} \approx L \omega_{c}=5.93 \Omega$ adjusts the bandwidth $\omega_{c}$, and the integral gain $k_{i}=k_{p} \omega_{z}=2981 \Omega \cdot \mathrm{s}^{-1}$ sets the PI's zero $\omega_{z}$ one decade below the bandwidth. The PR compensator is formed by adding resonant compensators $G_{R}(z)$ in parallel to the previous PI. Each resonant compensator can be implemented using an all-pass filter $G_{A P}(z)$ as [18]

$$
G_{R_{h}}(z)=\frac{G_{h}}{2}\left[1-G_{A P_{h}}(z)\right]
$$

being $h$ the harmonic number, $G_{h}$ the resonant gain and

$$
\begin{gathered}
G_{A P_{h}}(z)=\frac{k_{2} z^{2}+k_{1}\left(1+k_{2}\right) z+1}{z^{2}+k_{1}\left(1+k_{2}\right) z+k_{2}} \\
k_{1}=-\cos \left(\omega_{h} T\right) \\
k_{2}=\frac{1-\tan \left(B W_{h} T / 2\right)}{1+\tan \left(B W_{h} T / 2\right)}
\end{gathered}
$$


where $\omega_{h}=h \omega_{g}$ is the resonant frequency and $B W_{h}$ is the resonant bandwidth. The resonant compensators were tuned experimentally, as explained in [12].

Table 3. PI and PR control parameters.

\begin{tabular}{lcc}
\hline Description & Variable & Value \\
\hline Sampling period & $T$ & $50 \mu \mathrm{s}$ \\
Gain crossover frequency & $\omega_{c}$ & $2 \pi 800 \mathrm{rad} / \mathrm{s}$ \\
Proportional gain & $k_{p}$ & $5.93 \Omega$ \\
Integral gain & $k_{i}$ & $2981 \Omega \cdot \mathrm{s}^{-1}$ \\
2nd dq-harmonic resonant gain & $G_{2}$ & $40 \Omega$ \\
2nd dq-harmonic resonant bandwidth & $B W_{2}$ & $\pi \mathrm{rad} / \mathrm{s}$ \\
6th dq-harmonic resonant gain & $G_{6}$ & $60 \Omega$ \\
6th dq-harmonic resonant bandwidth & $B W_{6}$ & $2 \pi \mathrm{rad} / \mathrm{s}$ \\
12th dq-harmonic resonant gain & $G_{12}$ & $50 \Omega$ \\
12th dq-harmonic resonant bandwidth & $B W_{12}$ & $4 \pi \mathrm{rad} / \mathrm{s}$ \\
\hline
\end{tabular}

The resulting closed-loop frequency responses shown in Figure 9 indicate that the IPCC is a better choice to work with the CE method. While the PI and PR controls present a frequency dependent group delay of up to five samples, the IPCC presents a constant group delay of two samples. This constant group delay can be compensated by using a constant-size buffer-based method with filtering properties described in [12]. Figure 10 gives the line current THD variation with the number of estimation leading samples when using the IPCC, showing that an optimal delay compensation is achieved with four leading samples for all current levels.
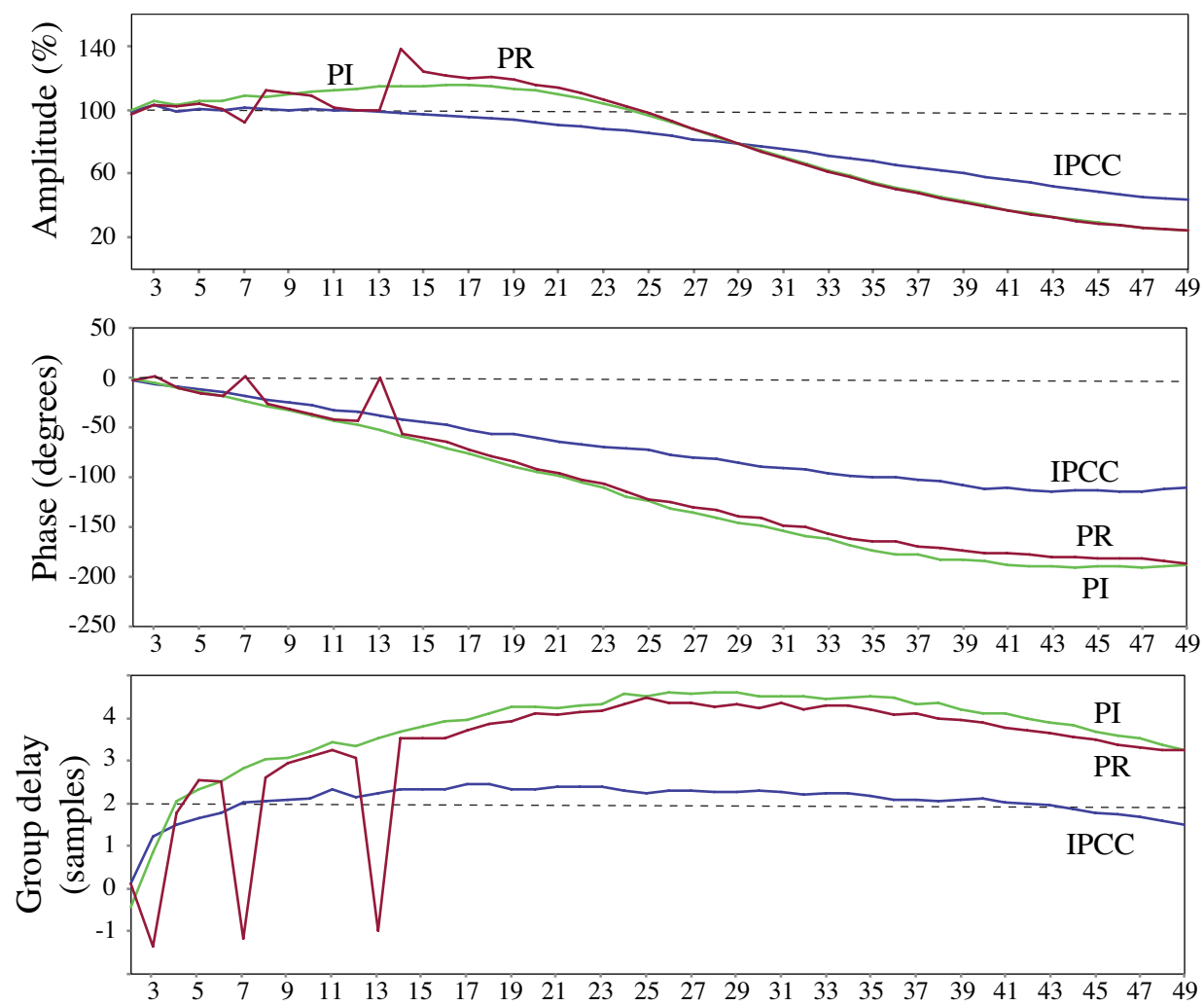

Harmonic number

Figure 9. Comparative experimental closed-loop Bode diagrams of IPCC, PI and PR controls. 


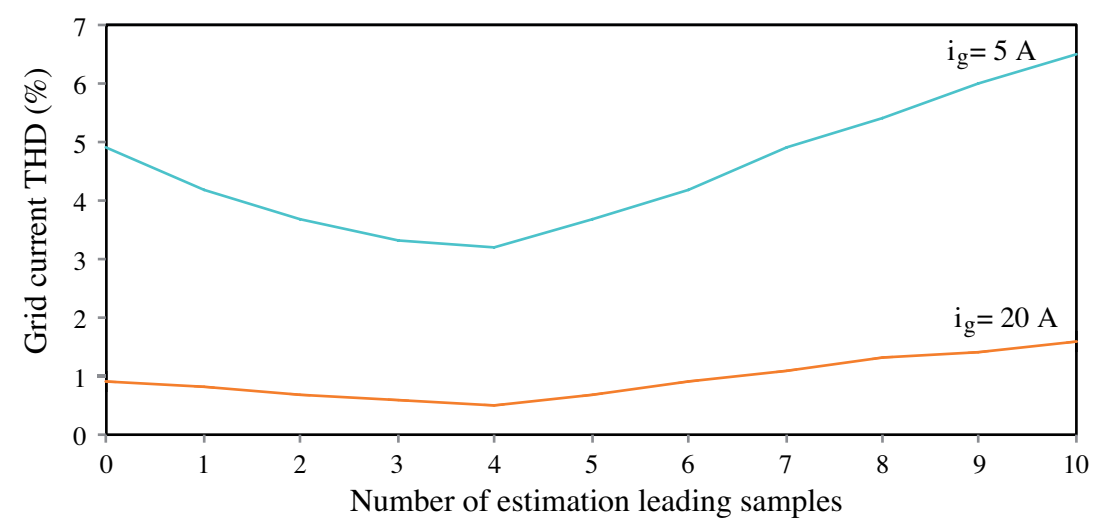

Figure 10. Line current THD as a function of the number of leading samples $n_{f}$ using the CE method with IPCC.

Figures 11 and 12 are intended to show the line current quality obtained with the combination CE + IPCC. The grid current waveform is shown in Figure 11a and its spectral density in Figure $11 \mathrm{~b}$ at full active power. The spectral density of the line currents varies with time but the $\mathrm{THD}_{i}$ remains between $0.3 \%$ and $0.5 \%$. The grid voltage distortion is $\mathrm{THD}_{v}=1.8 \%$, as can be seen in Figure $11 \mathrm{~b}$. All three phase grid currents at nominal power are also shown together in Figure 12.

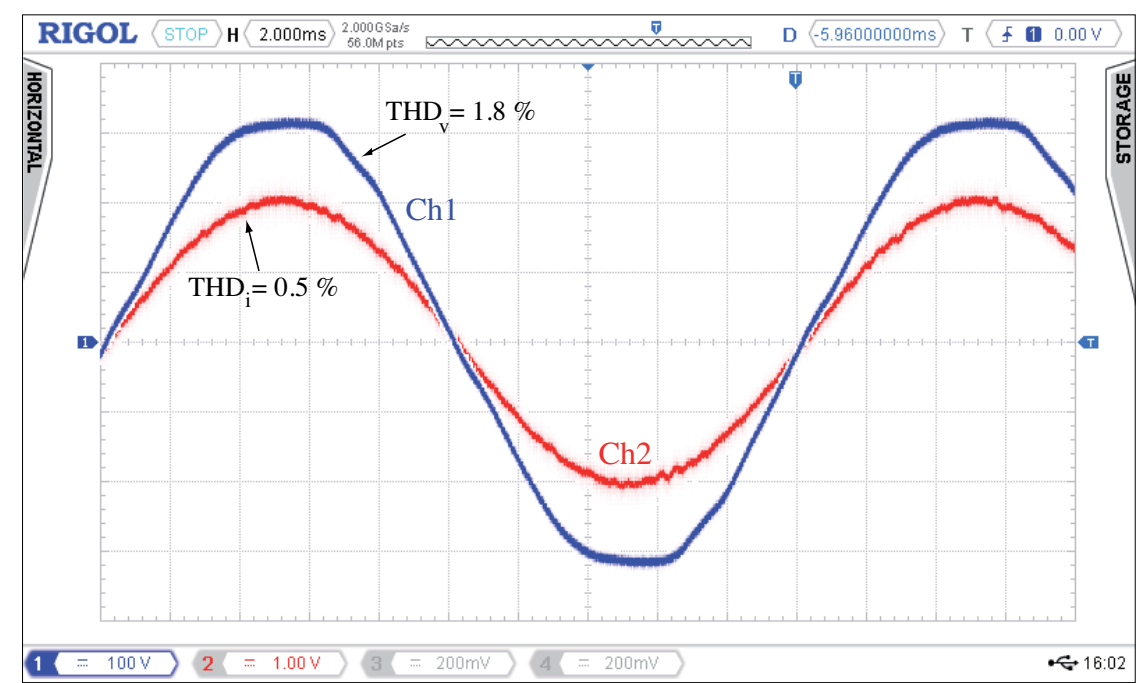

(a)

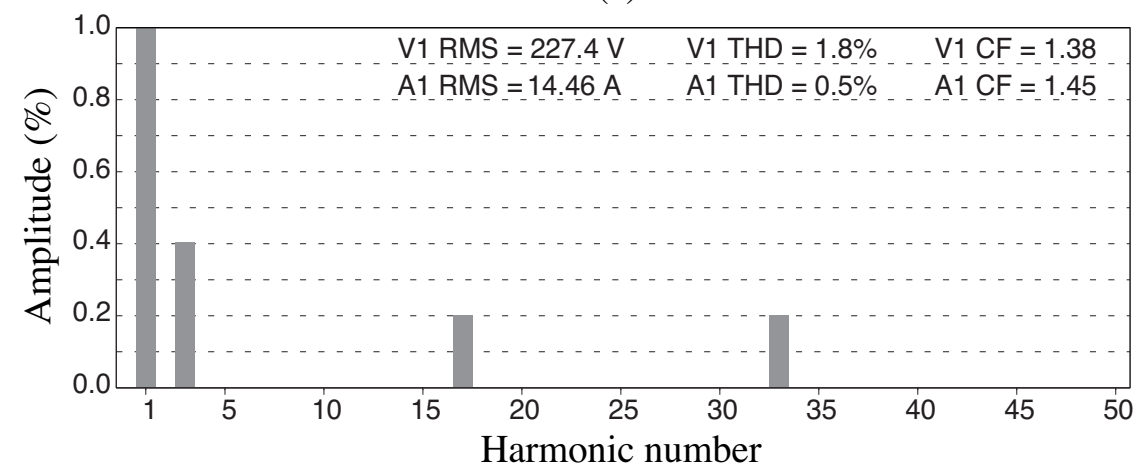

(b)

Figure 11. Grid current at nominal $10 \mathrm{kVA}$ using the IPCC control with CE: (a) grid voltage (Ch1: $100 \mathrm{~V} / \mathrm{div}$ ) and grid current (Ch2: $10 \mathrm{~A} / \mathrm{div})$; and (b) harmonic content of the grid current. 


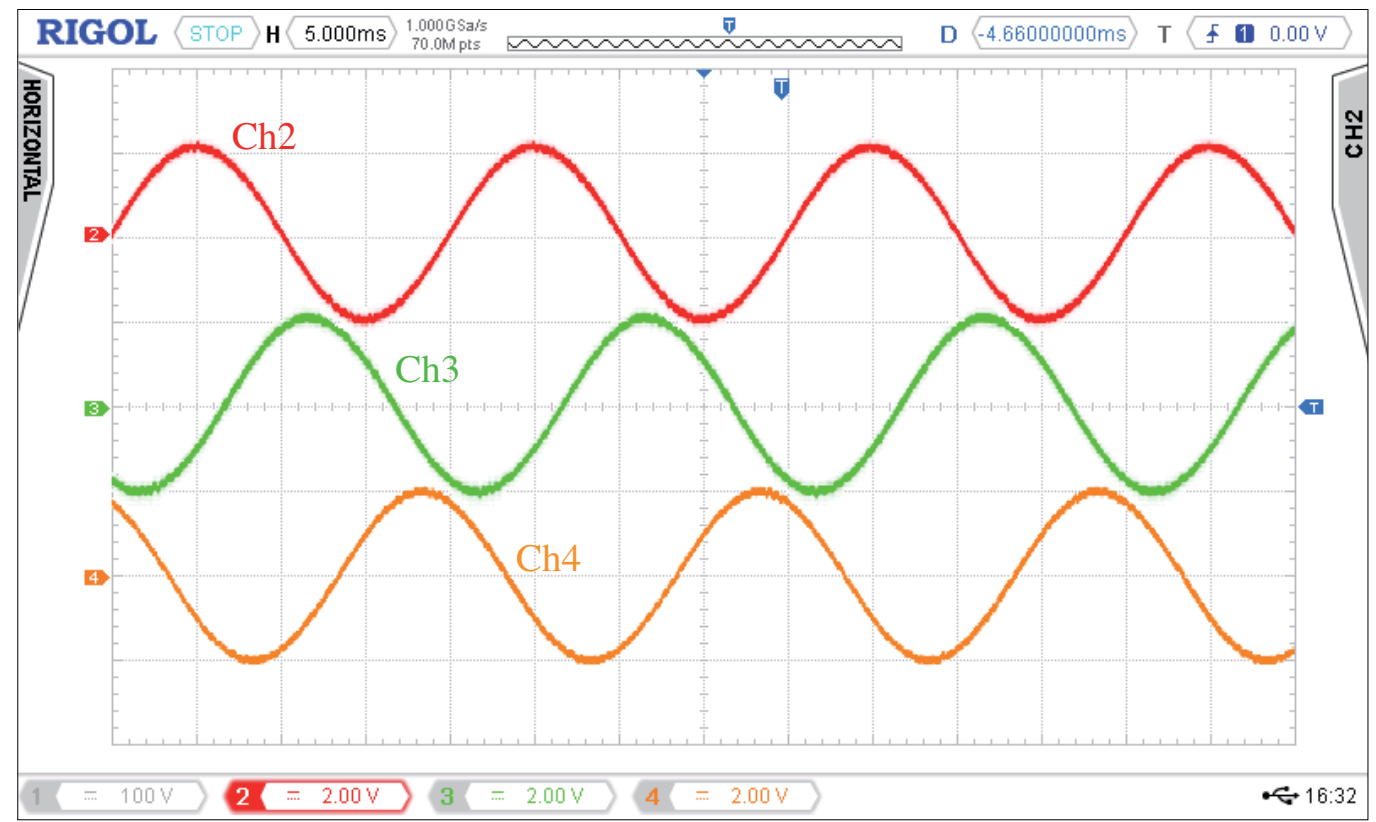

Figure 12. Grid currents at nominal $10 \mathrm{kVA}$ with the IPCC control and CE. Ch2: phase R (20 A/div); Ch3: phase S (20 A/div); Ch4: phase T (20 A/div).

Figure 13 illustrates the effect of using or not the CE strategy along with the IPCC control. At half the nominal power, the grid current $\mathrm{THD}_{i}$ is $4.0 \%$ without $\mathrm{CE}$, and it reduces to $1.5 \%$ with $\mathrm{CE}$.

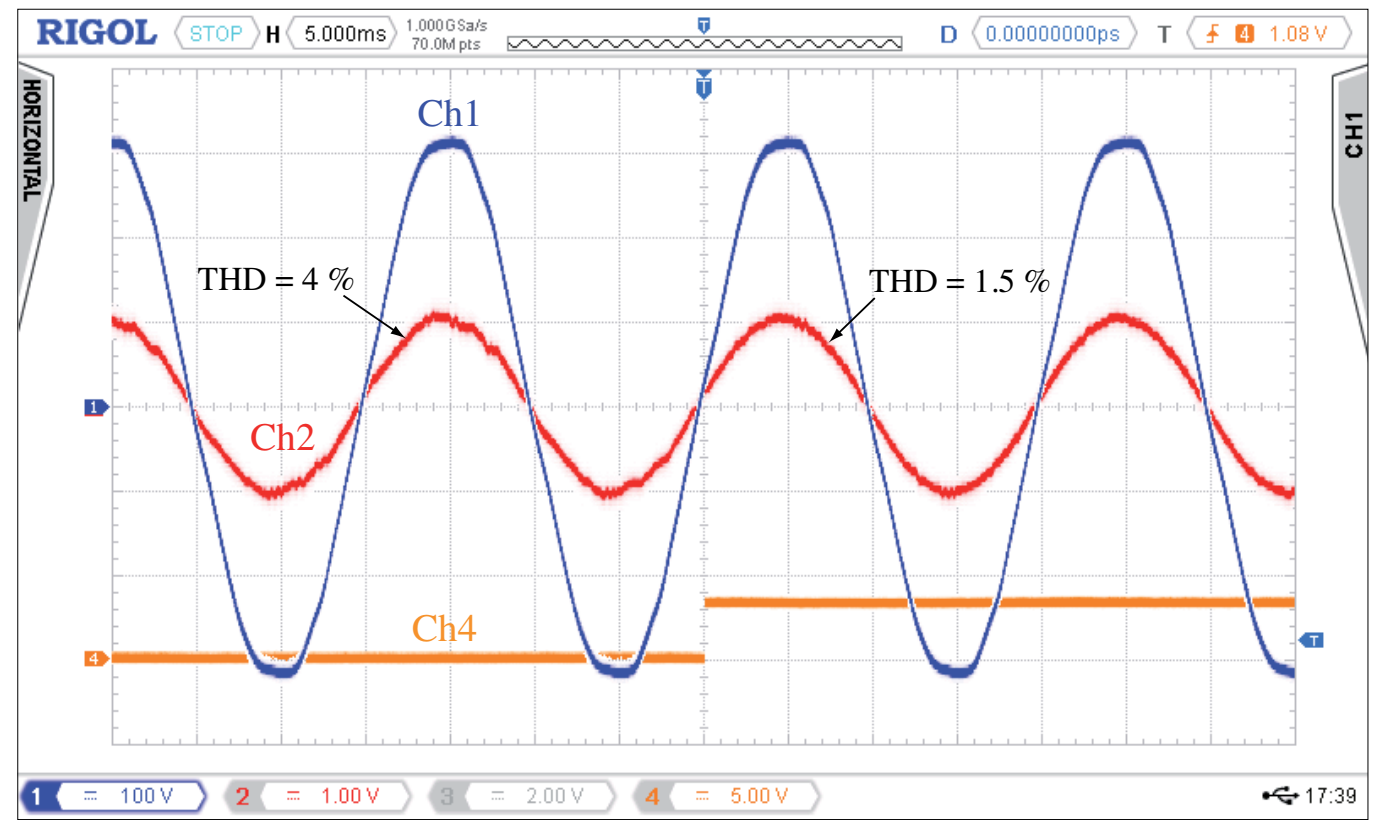

Figure 13. Activation-deactivation of the CE with IPCC control at half nominal power. Ch1: grid voltage (100 V/div); Ch2: grid current (10 A/div); Ch4: CE on/off signal $(0=$ off, $1=$ on).

Figure 14 shows the effects of using the $\mathrm{CE}$ technique on PR and IPCC controls. The CE effectiveness is better recognized when grid current (Ch2) is set to zero, as the inverter current (Ch4) just mimics the capacitor current (Ch3). While the PR in Figure 14a still exhibits some visible harmonics at zero grid current, the IPCC removes current harmonics, as shown in Figure 14b. When the current reference increases, the grid current distortion of the IPCC is noticeably smaller than that of the PR. 
To present a full comparative study, a GCF scheme was also implemented using the same previous PR control. Figure 15 summarizes the $\mathrm{THD}_{i}$ results for the five implemented control configurations as a function of the current amplitude. Notice that Cases $a$ and $e$ are also compared in Figure 13, and Cases $b$ and $e$ are compared in Figure 14. Although the GCF achieves very low $\operatorname{THD}_{i}$ levels around $1.4 \%$ at nominal power, even better than the CE + PR, best results are obtained with the proposed CE + IPCC, which lowers the $\mathrm{THD}_{i}$ down to $0.3-0.5 \%$, improving the $0.7 \%$ of the CE-PI option [12]. All measurements were carried out with a grid voltage distortion around $\mathrm{THD}_{v} \approx 2 \%$. It is important to remark that the transformerless setup is a more challenging test in terms of $\mathrm{THD}_{i}$ than the galvanically isolated setup, since the frequency applied to the LCL filter is higher in the isolated case.

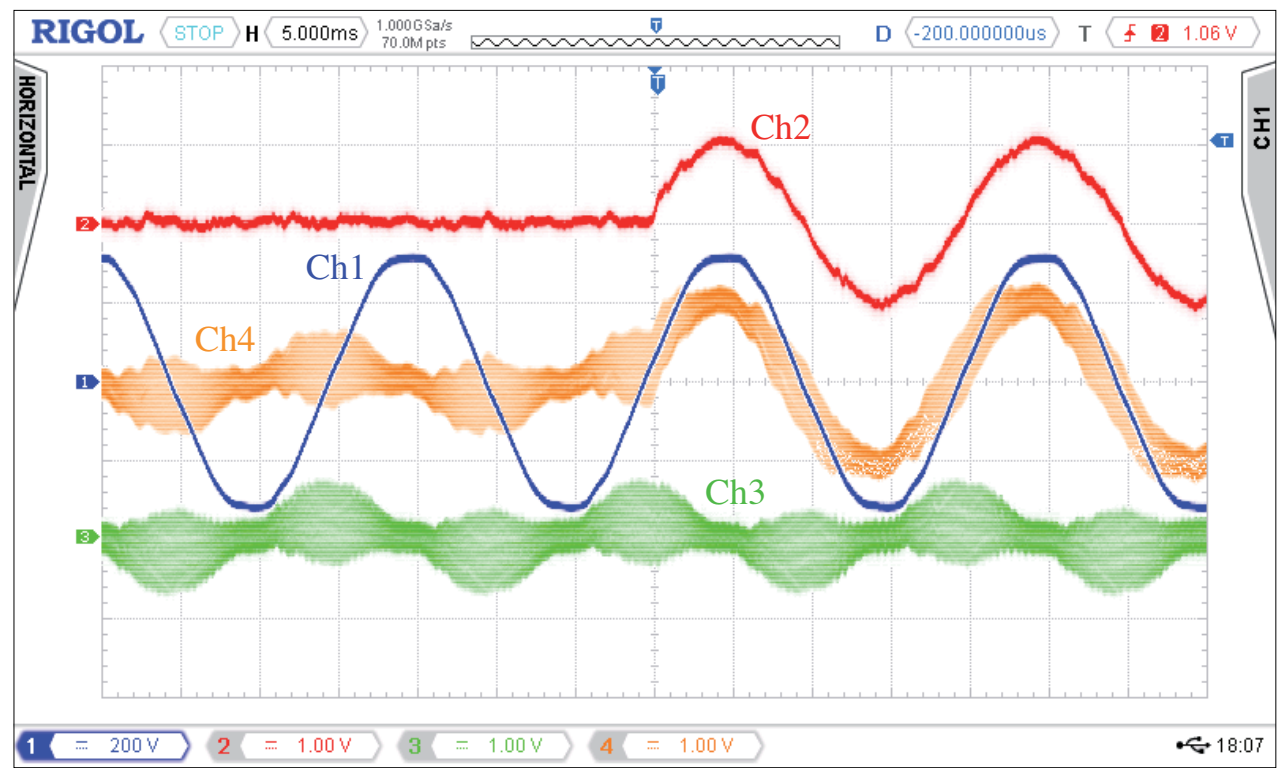

(a)

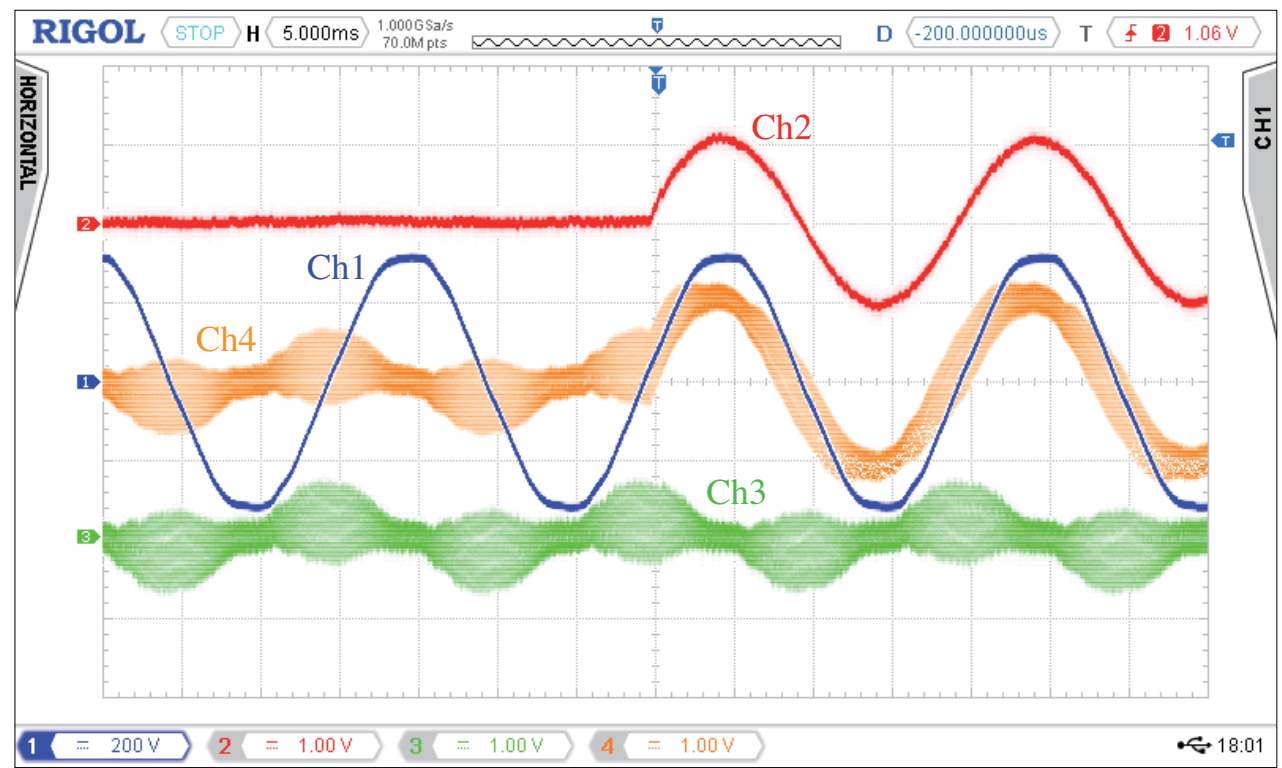

(b)

Figure 14. Comparison of the CE performance with: (a) PR control; and (b) IPCC control. Ch1: grid voltage (200 V/div); Ch2: grid current (10 A/div); Ch3: LCL capacitor current (10 A/div); Ch4: inverter current (10 A/div). 


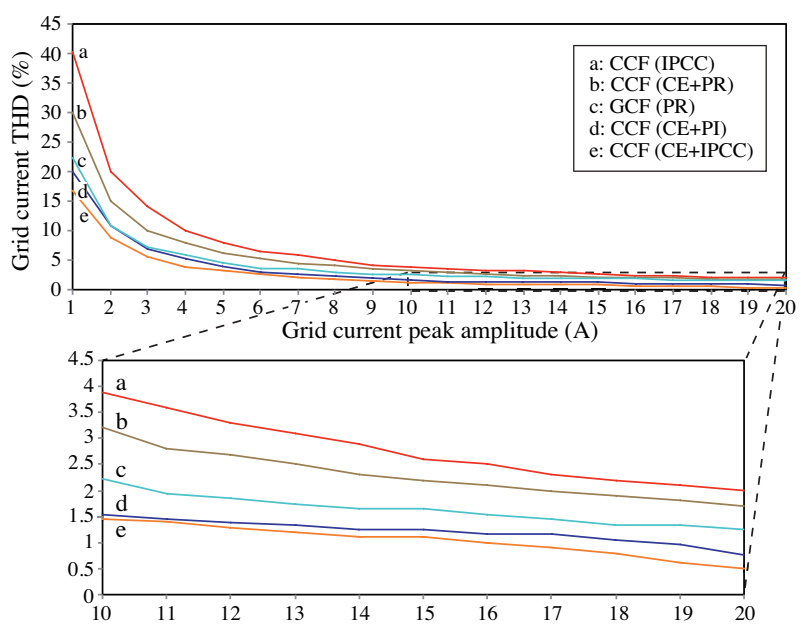

Figure 15. Grid current THD for different CCF (with and without the CE technique) and GCF strategies.

Finally, Figure 16 presents the dynamic performance of the proposed IPCC + CE against reference steps in amplitude $I_{0}$ and phase $\theta_{0}$. Figure 16a shows amplitude changes on the grid current produced by changes on the amplitude reference $I_{0}$ to $10 \mathrm{~A}, 15 \mathrm{~A}$ and $20 \mathrm{~A}$. It can be noticed that the amplitude levels are accurate, i.e., there is no current error, while presenting very fast transitions. On the other hand, Figure $16 \mathrm{~b}$ shows active and reactive power changes produced by reference angle $\theta_{0}$ steps in $0^{\circ}$, $-90^{\circ}$ and $90^{\circ}$.

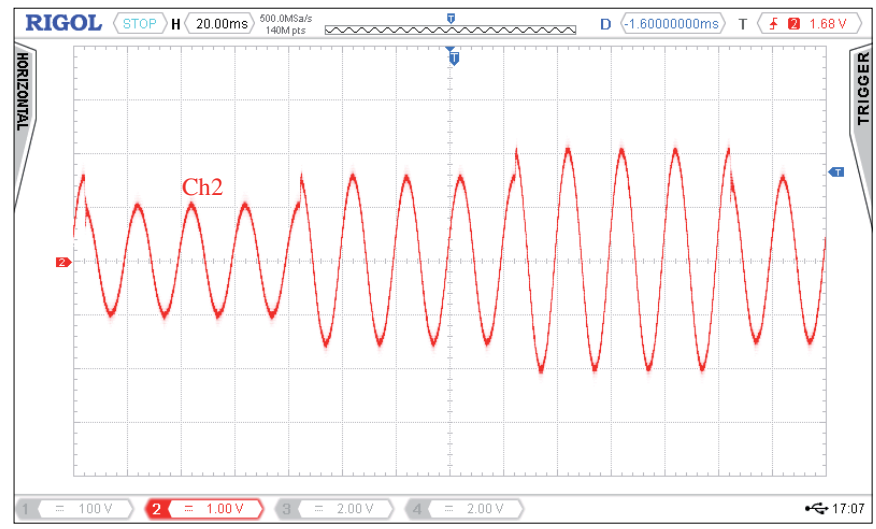

(a)

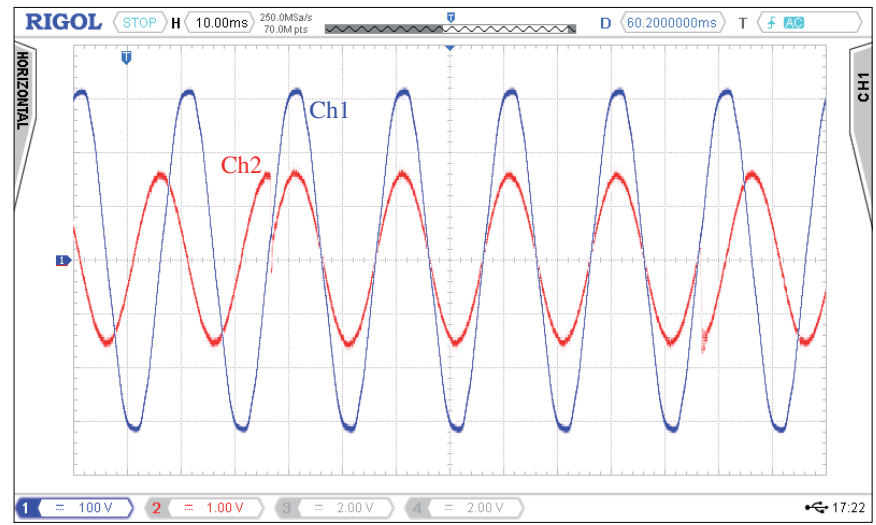

(b)

Figure 16. Grid current response of the IPCC + CE against reference variations in: (a) amplitude; and (b) phase. Ch1: grid voltage (100 V/div); Ch2: grid current (10 A/div). 


\section{Conclusions}

This paper combines the capacitive emulation (CE) technique with a new integral predictive current control (IPCC), which together significantly reduces the harmonic distortion of the line currents when using LCL filters and converter current feedback (CCF).

The proposed IPCC is derived from the low frequency model (below the $L_{2}-C$ resonance) of the LCL filter, combining a dead-beat algorithm with cascaded current observers and FIR filters to compensate for the total sensing delays. The IPCC adds integral action to remove the current error at the fundamental harmonic, without affecting the closed-loop dead-beat performance. A design equation for the integrator gain is provided as a function of the number of observers and the observer gain.

As shown in the experimental results, the IPCC control presents a constant group delay in closed-loop, unlike the PI and PR controls. This improves the control delay compensation of the CE method. Furthermore, the IPCC control presents a more accurate amplitude response for reference tracking compared with the PI and PR controls.

The effectiveness of the proposed solution has been proven experimentally on a $10 \mathrm{kVA}$ transformerless LCL-filtered inverter. Although the CE method can be used along with any other current control to reduce the line current distortion in a CCF scheme, the CE + IPCC combination achieves $\mathrm{THD}_{i}$ values around $0.3-0.5 \%$ at $10 \mathrm{kVA}$, even lower than a CE with a PI control [12] or a GCF scheme with a multi-resonant PR control.

Author Contributions: All authors contributed to the conceptualization, experimental validation and writing-review \& editing.

Conflicts of Interest: The authors declare no conflict of interest.

\section{References}

1. Pan, D.; Ruan, X.; Wang, X.; Yu, H.; Xing, Z. Analysis and design of current control schemes for LCL-type grid-connected inverter based on a general mathematical model. IEEE Trans. Power Electron. 2017, 32, 4395-4410.

2. Wang, J.; Yan, J.D.; Jiang, L. Pseudo-derivative-feedback current control for three-phase grid-connected inverters with lcl filters. IEEE Trans. Power Electron. 2016, 31, 3898-3912.

3. Wang, J.; Yan, J.D.; Jiang, L.; Zou, J. Delay-dependent stability of single-loop controlled grid-connected inverters with LCL filters. IEEE Trans. Power Electron. 2016, 31, 743-757.

4. Zhang, X.; Spencer, J.; Guerrero, J. Small-signal modeling of digitally controlled grid-connected inverters with LCL filters. IEEE Trans. Ind. Electron. 2013, 60, 3752-3765.

5. Jia, Y.; Zhao, J.; Fu, X. Direct grid current control of LCL-filtered grid-connected inverter mitigating grid voltage disturbance. IEEE Trans. Power Electron. 2014, 29, 1532-1541.

6. Bao, C.; Ruan, X.; Wang, X.; Li, W.; Pan, D.; Weng, K. Step-by-step controller design for LCL-type grid-connected inverter with capacitor-current-feedback active-damping. IEEE Trans. Power Electron. 2014, 29, 1239-1253.

7. Meyer, R.; Zlotnik, A.; Mertens, A. Fault ride-through control of medium-voltage converters with LCL filter in distributed generation systems. IEEE Trans. Ind. Appl. 2014, 50, 3448-3456.

8. Dannehl, J.; Wessels, C.; Fuchs, F. Limitations of voltage-oriented PI current control of grid-connected PWM rectifiers with LCL filters. IEEE Trans. Ind. Electron. 2009, 56, 380-388.

9. Tang, Y.; Loh, P.C.; Wang, P.; Choo, F.H.; Gao, F. Exploring inherent damping characteristic of LCL-filters for three-phase grid-connected voltage source inverters. IEEE Trans. Power Electron. 2012, 27, 1433-1443.

10. Ghoshal, A.; John, V. Active damping of LCL filter at low switching to resonance frequency ratio. IET Power Electron. 2015, 8, 574-582.

11. Citro, C.; Siano, P.; Cecati, C. Designing inverters' current controllers with resonance frequencies cancellation. IEEE Trans. Ind. Electron. 2016, 63, 3072-3080.

12. Espi, J.M.; Garcia-Gil, R.; Castello, J. Capacitive emulation for LCL-filtered grid-connected converters. Energies 2017, 10, 930 . 
13. Castello, J.; Espi, J.M.; Garcia-Gil, R. A new generalized robust predictive current control for grid-connected inverters compensates anti-aliasing filters delay. IEEE Trans. Ind. Electron. 2015, 63, 4485-4494.

14. Panten, N.; Hoffmann, N.; Fuchs, F.W. Finite control set model predictive current control for grid-connected voltage-source converters with LCL filters: A study based on different state feedbacks. IEEE Trans. Power Electron. 2016, 31, 5189-5200.

15. Donoso, F.; Mora, A.; Cárdenas, R.; Angulo, A.; Sáez, D.; Rivera, M. Finite-Set Model-Predictive Control strategies for a 3L-NPC inverter operating with fixed switching frequency. IEEE Trans. Ind. Electron. 2018, 65, 3954-3965.

16. Yan, Q.; Wu, X.; Yuan, X.; Geng, Y. An improved grid-voltage feedforward strategy for high-power three-phase grid-connected inverters based on the simplified repetitive predictor. IEEE Trans. Power Electron. 2016, 31, 3880-3897.

17. Rodriguez, P.; Luna, A.; Candela, I.; Mujal, R.; Teodorescu, R.; Blaabjerg, F. Multiresonant frequency-locked loop for grid synchronization of power converters under distorted grid conditions. IEEE Trans. Ind. Electron. 2011, 58, 127-138.

18. Gonzalez-Espin, F.; Garcera, G.; Patrao, I.; Figueres, E. An adaptive control system for three-phase photovoltaic inverters working in a polluted and variable frequency electric grid. IEEE Trans. Power Electron. 2012, 27, 4248-4261.

(C) 2018 by the authors. Licensee MDPI, Basel, Switzerland. This article is an open access article distributed under the terms and conditions of the Creative Commons Attribution (CC BY) license (http:/ / creativecommons.org/licenses/by/4.0/). 